\title{
On the Error Terms and Exceptional Sets in Conjectural Second Main Theorems
}

\author{
Aaron Levin \\ Centro di Ricerca Matematica Ennio De Giorgi \\ I-56100 Pisa, Italy \\ David McKinnon \\ Department of Pure Mathematics \\ University of Waterloo \\ Waterloo, ON, N2T 2M2 Canada * \\ Jörg Winkelmann \\ Institut für Mathematik \\ Universität Bayreuth \\ 95440 Bayreuth, Germany
}

\begin{abstract}
We study the error terms and exceptional sets in conjectural Second Main Theorems in Nevanlinna theory and Diophantine approximation (Vojta's conjecture). In particular, we give a geometric description of the exceptional set in the case of surfaces and the trivial divisor. Examples are given which show that, in general, the exceptional sets in conjectural Second Main Theorems must depend on the parameter $\epsilon$ in these conjectures. As a consequence, we obtain counterexamples to a conjecture of S. Lang on the form of the error term in conjectural Second Main Theorems.
\end{abstract}

\section{Introduction}

In 1925, R. Nevanlinna [11] proved his First and Second Main Theorems, thereby establishing a theory for the distribution of values of a meromorphic function $f: \mathbb{C} \rightarrow \mathbb{P}^{1}$. Generalizing Nevanlinna's Second Main Theorem to holomorphic curves in arbitrary complex varieties is a central open problem in Nevanlinna theory. It has been conjectured that a general Second Main Theorem holds in the following form.

${ }^{*}$ Research supported in part by Grant 250196-02 from the Natural Sciences and Engineering Research Council 
Conjecture 1. Let $X$ be a nonsingular complex projective variety, $D$ a normal crossings divisor on $X, K$ the canonical divisor on $X, A$ an ample divisor on $X$, and $\epsilon>0$. Then there exists a proper Zariski-closed subset $Z \subset X$, depending on the above data, such that for any non-constant holomorphic map $f: \mathbb{C} \rightarrow X$ with $f(\mathbb{C}) \not \subset Z$ we have

$$
m_{f, D}(r)+T_{f, K}(r) \leq \epsilon T_{f, A}(r)+O(1)
$$

for all $r$ outside a set of finite Lebesgue measure.

As first noticed by C. Osgood, certain statements in Nevanlinna theory strongly resemble certain statements in Diophantine approximation when viewed properly. A detailed Nevanlinna-Diophantine dictionary has been constructed by P. Vojta [14]. Under this correspondence, Nevanlinna's Second Main Theorem corresponds to Roth's theorem in Diophantine approximation. Inspired by his Nevanlinna-Diophantine dictionary and results in Nevanlinna theory, Vojta [14] made the following deep conjecture, an arithmetic counterpart to Conjecture 1 generalizing Roth's theorem.

Conjecture 2. Let $X$ be a nonsingular projective variety defined over a number field $k, S$ a finite set of places of $k$ containing the archimedean places, $D$ a normal crossings divisor on $X, K$ the canonical divisor on $X, A$ an ample divisor on $X$, and $\epsilon>0$. Then there exists a proper Zariski-closed subset $Z \subset X$, depending on the above data, such that for all points $P \in X(k) \backslash Z$,

$$
m_{D, S}(P)+h_{K}(P) \leq \epsilon h_{A}(P)+O(1) .
$$

We refer the reader to [14] for the basic definitions and properties of the objects appearing in Conjectures 1 and 2.

The set $Z$ in Conjecture 1 or Conjecture 2 is known as an exceptional set. Note that by increasing the $O(1)$ term in Conjecture 2 we can assume that every irreducible component of the exceptional set $Z$ has positive dimension. When $X=\mathbb{P}^{n}$ and $D$ is a sum of hyperplanes, Conjectures 1 and 2 are known to be true by theorems of Cartan and Schmidt, respectively, with the additional statement that $Z$ can be chosen to be a finite union of hyperplanes. Furthermore, in this situation, Vojta $[15,17]$ has shown that the exceptional set $Z$ can be chosen independent of $\epsilon$. It is thus natural to ask whether in general the exceptional set $Z$ can be chosen independent of $\epsilon$ in Conjectures 1 and 2 .

In addition to studying the exceptional set $Z$, we will be interested in the error terms $\epsilon T_{f, A}$ and $\epsilon h_{A}$ in Conjectures 1 and 2, respectively. In Cartan's theorem, the error term $\epsilon T_{f, A}$ has been sharpened to, for instance, $O(\log r+$ $\left.\log ^{+} T_{f, A}(r)\right)$. In [7, Conjecture VIII.5.1], S. Lang conjectured that a general Second Main Theorem should hold with this stronger error term:

Conjecture 3. Let $X$ be a nonsingular complex projective variety, $D$ a normal crossings divisor on $X, K$ the canonical divisor on $X$, and $A$ an ample divisor on $X$. Then there exists a proper Zariski-closed subset $Z \subset X$, depending on 
the above data, such that for any non-constant holomorphic map $f: \mathbb{C} \rightarrow X$ with $f(\mathbb{C}) \not \subset Z$ we have

$$
m_{f, D}(r)+T_{f, K}(r)+N_{f, R a m}(r) \leq O\left(\log r+\log ^{+} T_{f, A}(r)\right)
$$

for all $r$ outside a set of finite Lebesgue measure.

The purpose of this article is to give several examples which show:

A. There exist varieties $X$ for which the exceptional sets in Conjectures 1 and 2 must depend on $\epsilon$.

B. There exist counterexamples to Conjecture 3. There exist situations in which the error terms in Conjectures 1 and 2 are essentially best possible.

It is clear that (A) implies (B). For Vojta's generalization of Conjecture 2 to algebraic points [14, Conjecture 5.2.6], Vojta [15, Examples 8.13 and 8.15] has shown that the exceptional set must depend on $\epsilon$.

\section{Examples with $D=0$}

In this section we give examples with $D=0$ where the exceptional sets in Conjectures 1 and 2 must depend on $\epsilon$. Note that if Conjectures 1 or 2 hold in any given situation, then there is a (unique) minimal exceptional set $Z_{\text {min }}$ (with respect to set inclusion). We begin by giving a geometric description of the exceptional set for surfaces and $D=0$.

Theorem 4. Let $X$ be a nonsingular complex projective surface with canonical divisor $K$. Let $A$ be any ample divisor on $X$. Assume that Conjecture 1 is true for $X$. For any $\epsilon>0$, let $Z_{\min }(\epsilon)$ be the corresponding minimal exceptional set from Conjecture 1 with $D=0$. Then for irrational $\epsilon, Z_{\min }(\epsilon)$ is the union of the set of curves $C$ on $X$ satisfying the following two properties:

1. $C$ is a curve of geometric genus $g \leq 1$.

2. K.C> $>$ A.C.

Let $\mathcal{C}$ be the set of curves $C$ on $X$ satisfying property 1 and K.C>0. Then the exceptional set $Z$ in Conjecture 1 (for $X, A$, and $D=0$ ) can be chosen independent of $\epsilon$ if and only if the set $\mathcal{C}$ is finite.

Proof. Let $C$ be a curve satisfying 1 and 2 . Let $\phi: \tilde{C} \rightarrow \underset{\tilde{C}}{C} \rightarrow X$ be the natural map obtained from the normalization $\tilde{C}$ of $C$. Since $g(\tilde{C}) \leq 1$, there exists a non-constant holomorphic map $f: \mathbb{C} \rightarrow \tilde{C}$. Let $P$ be a point on $\tilde{C}$ (viewed as a divisor of degree 1). By functoriality and quasi-equivalence of the characteristic functions of numerically equivalent divisors, we have, for any $\delta>0$,

$$
T_{\phi \circ f, K}(r)>(K . C-\delta) T_{f, P}(r)
$$


and

$$
T_{\phi \circ f, A}(r)<(A . C+\delta) T_{f, P}(r)
$$

as $r \rightarrow \infty$. Since $0<\epsilon<\frac{K . C}{A . C}$, it follows that

$$
T_{\phi \circ f, K}(r)>\epsilon T_{\phi \circ f, A}(r)
$$

as $r \rightarrow \infty$. Therefore $C$ belongs to the exceptional set $Z_{\min }(\epsilon)$ of Conjecture 1 , as desired.

Conversely, if $C$ is a curve which is a component of $Z_{\min }(\epsilon)$, then we have

$$
T_{f, K}(r)>\epsilon T_{f, A}(r)+O(1)
$$

as $r \rightarrow \infty$ for some non-constant holomorphic map $f: \mathbb{C} \rightarrow C$. Since $C$ admits a non-constant holomorphic map, by a theorem of Picard $C$ must have geometric genus at most one. Reversing the above arguments, we also see that we must have $K . C \geq \epsilon A$.C. Since $\epsilon$ is irrational, this implies the strict inequality $K . C>$ $\epsilon A$. $C$ (the only reason we assumed $\epsilon$ to be irrational was to avoid consideration of the boundary case $K . C=\epsilon A . C$ ).

If the set $\mathcal{C}$ of curves $C$ with geometric genus $g \leq 1$ and $K . C>0$ is infinite, then by the above, $Z(\epsilon)$ cannot be a fixed proper Zariski-closed subset of $X$ as $\epsilon \rightarrow 0$. Conversely, if $\mathcal{C}$ is finite, then we may take $Z=\cup_{C \in \mathcal{C}} C$ in Conjecture 1, independent of $\epsilon$.

Theorem 5. Let $X$ be a nonsingular projective surface defined over a number field $k$ with canonical divisor $K$. Let $A$ be any ample divisor on $X$ defined over $k$. Assume that Conjecture 2 is true for $X$. For any $\epsilon>0$, let $Z_{\min }(\epsilon)$ be the corresponding minimal exceptional set from Conjecture 2 over $k$ with $D=0$. Then for irrational $\epsilon, Z_{\min }(\epsilon)$ is the union of the curves $C$ on $X$ satisfying the following two properties:

1. $C$ contains infinitely many $k$-rational points.

2. $K . C>\epsilon A . C$.

Let $\mathcal{C}$ be the set of curves $C$ on $X$ satisfying property 1 and $K . C>0$. Then the exceptional set $Z$ in Conjecture $2($ for $X, A, k$, and $D=0$ ) can be chosen independent of $\epsilon$ if and only if the set $\mathcal{C}$ is finite.

Proof. Let $C$ be a curve satisfying 1 and 2. Using the appropriate analogous properties of heights, the proof of Theorem 4 shows that for $\epsilon<\frac{K . C}{A . C}$,

$$
h_{K}(P)>\epsilon h_{A}(P)
$$

for infinitely many $P \in C(k)$. So $C$ belongs to the exceptional set $Z_{\min }(\epsilon)$ of Conjecture 2 as soon as $\epsilon<\frac{K . C}{A . C}$.

Conversely, if $C$ is a component of $Z_{\min }(\epsilon)$, then $C$ must contain an infinite set of $k$-rational points, and for infinitely many of those points we have

$$
h_{K}(P)>\epsilon h_{A}(P)+O(1) .
$$


Pulling everything back to $C$ as in the proof of Theorem 4, we conclude from this that $K . C>\epsilon A$. $C$, as desired.

Let $\mathcal{C}$ be the set of curves $C$ with infinitely many $k$-rational points and $K . C>0$. If $\mathcal{C}$ is infinite, then by the above, $Z(\epsilon)$ cannot be a fixed proper Zariski-closed subset of $X$ as $\epsilon \rightarrow 0$. Conversely, if $\mathcal{C}$ is finite, then we may take $Z=\cup_{C \in \mathcal{C}} C$ in Conjecture 2 (over $k$ ), independent of $\epsilon$.

By Faltings' theorem, condition 1 in Theorem 5 implies that $C$ has geometric genus zero or one. Notice that Theorems 4 and 5 partially generalize to varieties $X$ of arbitrary dimension; if a curve $C$ on $X$ satisfies 1 and 2 of Theorem 4 (resp. Theorem 5), then $C$ belongs to the exceptional set $Z_{\min }(\epsilon)$ associated to Conjecture 1 (resp. Conjecture 2). Of course, if $X$ has dimension greater than two, $Z_{\min }(\epsilon)$ might contain components other than curves, so in general the above curves might not completely describe the exceptional set. Nevertheless, should an infinite set of curves $\mathcal{C}$ on $X$ exist with the given properties, then provided the union of the curves is Zariski-dense in $X$, the exceptional set $Z(\epsilon)$ must depend on $\epsilon$.

Note that if the set $\mathcal{C}$ in Theorem 5 is infinite for some $X$ and $k$, then the set $\mathcal{C}$ in Theorem 4 for $X$ will also be infinite. We give two examples of surfaces for which the set $\mathcal{C}$ of Theorem 5 is infinite for some $k$. Thus, for these surfaces, the exceptional sets in Conjectures 1 and 2 must depend on $\epsilon$.

Example 6. (The following example is implicit in the proof of the main theorem of [8].) Let $C$ be an elliptic curve defined over a number field $k$, and assume that $C$ has positive Mordell-Weil rank over $k$. For any pair of relatively prime integers $a$ and $b$, there is an irreducible curve $C_{a, b}$ on $C \times C$ defined by $C_{a, b}=$ $\{(P, Q) \mid a P=b Q\}$. Since $C$ has positive Mordell-Weil rank over $k$, every $C_{a, b}$ has infinitely many $k$-rational points. Let $X$ be the blowup of $C \times C$ at the origin $(O, O)$, with exceptional divisor $E$. The canonical divisor of $X$ is just $E$, since the canonical divisor of $C \times C$ is trivial. Let $C_{a, b}^{\prime}$ denote the strict transform of $C_{a, b}$ on $X$. As every curve $C_{a, b}$ passes through $(0,0)$ on $C \times C$, we have $K . C_{a, b}^{\prime}>0$. Therefore the set $\mathcal{C}$ of Theorem 5 contains the infinite set $\left\{C_{a, b}^{\prime} \mid(a, b)=1\right\}$ of curves on $X$.

Example 7. (This example stems from work of Kodaira and Nagata [10] on surfaces with infinitely many exceptional curves of the first kind; see Example 14 and the discussion leading up to it. It was observed independently by Vojta [16] and by the third author.)

Let $X_{0}$ be a nonsingular projective elliptic surface over $\mathbb{P}_{\mathbb{Q}}^{1}$ with positive Mordell-Weil rank (an example of such a surface will be given below). Applying the adjunction formula to a smooth fiber, we see that the canonical divisor $K_{0}$ can be written as a linear combination of fiber components. Therefore there exists an integer $m$ such that $K_{0} \geq-m F$ relative to the cone of effective divisors, where $F$ denotes a fiber of the elliptic fibration.

By induction on $m$, we may show that there exists a section of infinite order which meets the zero section in at least $m+1$ distinct places of good reduction. This is using the facts that (a) if a section $s$ meets the zero section at a given 
place $v$ of good reduction, then so do all multiples of $s$; and (b) if $s$ has infinite order then some nonzero multiple of $s$ must meet the zero section at a strictly larger set of places (except possibly for places of bad reduction). (This latter fact follows from Siegel's theorem.)

Given such a section $s$, let $\pi: X \rightarrow X_{0}$ be the blowing-up of $X_{0}$ along those $m+1$ points of intersection. Let $E_{1}, \ldots, E_{m+1}$ be the resulting exceptional divisors; then the canonical divisor $K$ of $X$ satisfies $K=\pi^{*} K_{0}+E_{1}+\cdots+E_{m+1}$. On $X_{0}$ we have $\left([u] s . K_{0}\right) \geq-m$ for all $u \in \mathbb{Z}$; therefore since all $[u] s$ meet all $E_{i}$ on $X$, we have

$$
([u] s . K) \geq 1
$$

on $X$. Moreover these are distinct rational curves on $X$, each having at least one rational point over $\mathbb{Q}$. Therefore each such curve has infinitely many rational points over $\mathbb{Q}$. So the infinite set of curves $\{[u] s \mid u \in \mathbb{Z}\}$ on $X$ is contained in the set $\mathcal{C}$ of Theorem 5 (with $k=\mathbb{Q}$ ).

To complete the example, consider the elliptic fibration

$$
y^{2}=4 x^{3}-3 t(t-1) x-t^{2}(t-1) .
$$

This is a surface of degree three, so its projective closure is rational. To see that its Mordell-Weil group has positive rank, we may either note that it does not appear in the list in [9] (the $j$-invariant has the wrong degree), or we may observe directly that the section $(t, \pm 2 t)$ has infinite order (specialize to $t=-1$ and use Mazur's theorem on $E(\mathbb{Q})_{\text {tors }}$ ).

In both Example 6 and Example 7, since the degrees of the given curves become large, only finitely many of the curves will be in the exceptional set for any given $\epsilon>0$.

\section{Dirichlet Approximation and More Examples}

Continuing our analysis of the exceptional sets in Conjectures 1 and 2, we provide a sufficient condition for $Z(\epsilon)$ to necessarily depend on $\epsilon$ when $X$ is a surface and $D$ is chosen to be sufficiently ample.

Theorem 8. Let $X$ be a nonsingular complex projective surface with canonical divisor $K$. Suppose that there exists an infinite set $\mathcal{C}$ of curves on $X$ such that every curve $C$ in $\mathcal{C}$ has the following two properties:

1. $C$ is a rational curve.

2. $K . C \geq-1$.

Then there exists a normal crossings divisor $D$ on $X$ such that for any ample divisor $A$ on $X$ the exceptional set $Z(\epsilon)$ in Conjecture 1 (for $X, A$, and $D$ ) must depend on $\epsilon$. 
Proof. Let $E$ be a very ample divisor on $X$. By Bertini's theorem, there exists a nonsingular irreducible divisor $D$ on $X$ with $D \sim 2 E$. In particular, $D$ has normal crossings. Let $C \in \mathcal{C}, C \neq D$. Let $\phi: \mathbb{P}^{1} \rightarrow C \rightarrow X$ be the normalization map composed with the inclusion map. Since $D \sim 2 E$ and $E$ is very ample, $\phi^{*} D$ consists of at least two points $P$ and $Q$ (counting multiplicities, so possibly $P=Q)$. Composing the exponential map with an automorphism of $\mathbb{P}^{1}$ sending $P$ and $Q$ to 0 and $\infty$, respectively, (or just sending $P$ to $\infty$ if $P=Q$ ), we obtain a non-constant holomorphic map $f: \mathbb{C} \rightarrow \mathbb{P}^{1}$ whose image omits $P$ and $Q$. Let $A$ be an ample divisor on $X$. By elementary properties, we deduce

$$
\liminf _{r \rightarrow \infty} \frac{m_{\phi \circ f, D}(r)}{T_{\phi \circ f, A}(r)} \geq \frac{2}{A . C} .
$$

Similarly, using $K . C \geq-1$, we obtain

$$
\liminf _{r \rightarrow \infty} \frac{m_{\phi \circ f, D}(r)+T_{\phi \circ f, K}(r)}{T_{\phi \circ f, A}(r)} \geq \frac{1}{A . C} .
$$

Therefore $C$ belongs to the exceptional set $Z(\epsilon)$ of Conjecture 1 as soon as $\epsilon<\frac{1}{A . C}$. Since $\mathcal{C}$ is infinite, the exceptional set must therefore depend on $\epsilon$.

To prove the arithmetic counterpart to Theorem 8 we need a version of Dirichlet's approximation theorem. The exponential function exp : $\mathbb{C} \rightarrow \mathbb{C} \subset \mathbb{P}^{1}$ omits as many points of $\mathbb{P}^{1}$ as possible for a holomorphic map (i.e., 2). The Diophantine analogue for this is a sequence of rational points which gives a good approximation to a given point $\alpha$. In the case $k=\mathbb{Q}$, such a sequence is provided by the following classical theorem of Dirichlet.

Theorem 9 (Dirichlet). Let $\alpha \in \mathbb{R} \backslash \mathbb{Q}$. Then there exist infinitely many pairs of coprime integers $(p, q)$ with

$$
\left|\alpha-\frac{p}{q}\right|<\frac{1}{q^{2}} .
$$

This can be generalized to a number field $k$ with a complex place $v$ as follows [12, pp. 253-255]:

Theorem 10. Let $k$ be a number field with a complex place $v$. Let $\alpha \in \mathbb{C} \backslash k$. Let $H_{k}$ be the multiplicative height on $k$. Then there exists a positive constant $C$ and infinitely many elements $\beta \in k$ with

$$
|\alpha-\beta|_{v}^{2}<\frac{C}{H_{k}(\beta)^{2}} .
$$

From this we obtain the following corollary.

Corollary 11. Let $k$ be a number field with a complex place $v$ and degree $[k$ : $\mathbb{Q}]>2$. Let $S$ be a finite set of places of $k$ containing the archimedean places. Let $D$ be an effective divisor of degree two or more on $\mathbb{P}^{1}$ (defined over some 
extension of $k$ ) and let $A$ be an ample divisor on $\mathbb{P}^{1}$. Then there exists a constant $C>0$ and infinitely many $k$-rational points $x \in \mathbb{P}^{1}(k)$ such that

$$
m_{D, S}(x)>2 \frac{h_{A}(x)}{\operatorname{deg}(A)}-C .
$$

Proof. Assume first that $D \backslash \mathbb{P}^{1}(k) \neq \emptyset$. Let $\alpha \in D \backslash \mathbb{P}^{1}(k)$. Looking only at the complex place $v$ of $k$, Theorem 10 immediately gives, upon taking logarithms, that there exists a constant $C>0$ and infinitely many points $x \in P^{1}(k)$ with

$$
m_{D, S}(x)>2 h(x)-C,
$$

where $h$ is the absolute logarithmic height on $\mathbb{P}^{1}$. Since $h(x)=\frac{h_{A}(x)}{\operatorname{deg}(A)}+O(1)$, we are done in this case.

Now if $D \backslash \mathbb{P}^{1}(k)=\emptyset$, then $D$ contains two $k$-rational points $P$ and $Q$ (counted with multiplicities). After a $k$-automorphism of $\mathbb{P}^{1}$ we may assume that either $P=0$ and $Q=\infty$ or $P=Q=\infty$. In this case, for the infinite set (since $[k: \mathbb{Q}]>2) R=\left\{(u, v) \in \mathbb{P}^{1} \mid u, v \in \mathcal{O}_{k}^{*}\right\}$, we have

$$
m_{D, S}(x) \geq m_{P+Q, S}(x)=2 h(x)+O(1)=2 \frac{h_{A}(x)}{\operatorname{deg}(A)}+O(1)
$$

for all $x \in R$ (in other words, $R$ is a set of $S$-integral points on $\mathbb{P}^{1} \backslash\{P, Q\}$ ).

We now prove the arithmetic analogue of Theorem 8 .

Theorem 12. Let $X$ be a nonsingular projective surface defined over a number field $k$ with canonical divisor $K$. Suppose that there exists an infinite set $\mathcal{C}$ of curves on $X$, all defined over $k$, such that every curve $C$ in $\mathcal{C}$ has the following two properties:

1. $C$ is a rational curve with infinitely many $k$-rational points.

2. $K . C \geq-1$.

Then there exists a normal crossings divisor $D$ on $X$ such that for any ample divisor $A$ on $X$ the exceptional set $Z(\epsilon)$ in Conjecture 2 (for $X, A, k$, and $D$ ) must depend on $\epsilon$.

Proof. After enlarging $k$, we can assume that $k$ has a complex place $v$ and that $[k: \mathbb{Q}]>2$. Let $E$ be a very ample divisor on $X$ defined over $k$. By Bertini's theorem, there exists a nonsingular irreducible divisor $D$ on $X$, defined over $k$, with $D \sim 2 E$. Let $C \in \mathcal{C}, C \neq D$. Let $\phi: \mathbb{P}^{1} \rightarrow C \rightarrow X$ be the normalization map, defined over $k$, composed with the inclusion map. Since $D \sim 2 E, \phi^{*} D$ is an effective divisor of degree two or more. Let $A$ be an ample divisor on $X$. By Corollary 11 , there are infinitely many $k$-rational points $P$ on $\mathbb{P}^{1}$ with

$$
m_{\phi^{*} D}(P)>\frac{2 h_{\phi^{*} A}(P)}{A . C}+O(1)
$$


or by functoriality,

$$
m_{D}(\phi(P))>\frac{2 h_{A}(\phi(P))}{A . C}+O(1)
$$

Using $K . C \geq-1$, we obtain $h_{K}(P)>-\frac{h_{A}(P)}{A \cdot C}+O(1)$ for $P \in C(k)$. So

$$
m_{D}(P)+h_{K}(P)>\frac{h_{A}(P)}{A . C}+O(1)
$$

for infinitely many $P \in C(k)$. Therefore $C$ belongs to the exceptional set $Z(\epsilon)$ of Conjecture 2 as soon as $\epsilon<\frac{1}{A . C}$. Since $\mathcal{C}$ is infinite, the exceptional set must therefore depend on $\epsilon$.

Similar to the $D=0$ case, Theorems 8 and 12 can be extended to varieties of arbitrary dimension, provided that one adds the hypothesis that the union of the curves in $\mathcal{C}$ is Zariski-dense in $X$.

We now give two examples of varieties which contain an infinite set $\mathcal{C}$ satisfying the hypotheses of Theorems 8 and 12. This provides more examples of situations where the exceptional sets in Conjectures 1 and 2 must depend on $\epsilon$.

Example 13 (In the case of holomorphic curves, this example is essentially Remark 0.3 on page 106 of [6]). Let $X$ be a smooth K3 surface defined over a number field $k$, such that $X$ contains infinitely many rational curves $C$ each with infinitely many $k$-rational points. For instance, the Kummer surface associated to the self-product $E \times E$ of an elliptic curve $E$ over $k$ will be such a K3 surface. Indeed, it contains the images of the curves $C_{a b}=\{(P, Q) \in E \times E \mid a P=b Q\}$ for coprime integers $a$ and $b$, and these images are all rational curves with infinitely many $k$-rational points. (For more examples of K3 surfaces with the desired property, see Theorem 4.10 of [3].) Since the canonical class of a K3 surface is trivial, $K . C=0$ for any such curve $C$.

Let $X$ be a smooth complex projective surface. An exceptional curve of the first kind on $X$ is an irreducible curve $C$ on $X$ with $C . C=C . K_{X}=-1$ (see e.g. [2]). Exceptional curves of the first kind may also be characterized by the property that there is a surjective morphism from $X$ onto a smooth projective surface $X_{1}$ which maps $C$ to a point and is an isomorphism outside $C$. Every exceptional curve of the first kind is a smooth rational curve. Therefore any such curve $C$ satisfies properties 1 and 2 of Theorem 8. If $C$ and $X$ are defined over a number field $k$, then $C$ also satisfies properties 1 and 2 of Theorem 12. Hence, any surface defined over a number field $k$ with infinitely many exceptional curves of the first kind defined over $k$ provides an example of the desired type. We give such a surface in Example 14.

Exceptional curves are rigid, i.e., there are no continuous families of them. Thus a projective surface $X$ contains at most countably infinitely many such curves. An argument of Kodaira, published by Nagata ([10], see also [5], ex. V.4.15), shows that there do exist surfaces with infinitely many exceptional curves of the first kind. The proof of Kodaira shows that such a surface can be 
obtained by blowing up at least nine points on $\mathbb{P}^{2}$ in very general position (i.e., outside of a countable union of proper subvarieties of $\left.\left(\mathbb{P}^{2}\right)^{9}\right)$.

In the arithmetic context "very general" is vacuous since there are only countably many rational points for any variety defined over a number field. For this reason we do not use Kodaira's original construction. Rather we follow an idea which we learned from Frederic Campana.

Example 14. Let $k$ be a number field and $E$ an elliptic curve over $k$ such that the group of $k$-rational points $E(k)$ has a non-torsion element $\alpha$. (E.g., we may take $k=\mathbb{Q}$ and $E \subset \mathbb{P}^{2}$ defined by $z y^{2}+y z^{2}=x^{3}+z^{2} x$, see [5, p. 28].)

We assume $E$ is embedded into $\mathbb{P}^{2}$ as a smooth cubic $k$-curve and denote the associated line bundle over $\mathbb{P}^{2}$ by $L$. Denoting the ideal sheaf of $E$ by $\mathcal{I}$ and the sheaf of sections in $L$ by $\mathcal{L}$ we have a short exact sequence of coherent sheaves

$$
\left.0 \rightarrow \mathcal{I} \otimes \mathcal{L} \rightarrow \mathcal{L} \rightarrow \mathcal{L}\right|_{E} \rightarrow 0 .
$$

Note that $\mathcal{I} \otimes \mathcal{L} \simeq \mathcal{O}$ and $H^{1}\left(\mathbb{P}^{2}, \mathcal{O}\right)=0$. Therefore the associated long exact sequence of cohomology groups yields

$$
0 \rightarrow \sigma \mathbb{C} \rightarrow \Gamma\left(\mathbb{P}^{2}, L\right) \rightarrow \Gamma(E, L) \rightarrow 0
$$

where $\sigma$ is a section of $L$ vanishing precisely on $E$. Thus the theorem of Abel implies that for any choice of eight points $p_{1}, \ldots, p_{8} \in E$ there is a ninth point $p_{9} \in E$ and a section $s \in \Gamma\left(\mathbb{P}^{2}, L\right)$ such that $\left\{p_{1}, \ldots, p_{9}\right\}$ is the zero-locus of $\left.s\right|_{E}$. We choose $p_{1}, \ldots, p_{9}$ in such a way with the additional conditions that all the $p_{i}$ are $k$-rational points, $p_{1}$ is the neutral element for the group law, and $p_{2}$ defines an element of infinite order with respect to the group law on the elliptic curve $E$. Next we blow up all the nine points $p_{i}$ and obtain a surface $X$, again defined over $k$. By the preceding cohomology calculations we know that the vector space

$$
\left\{\tau \in \Gamma\left(\mathbb{P}^{2}, L\right) \mid \tau\left(p_{i}\right)=0 \forall i=1, \ldots, 9\right\}
$$

is a two-dimensional vector space. The corresponding linear system consists of cubics on $\mathbb{P}^{2}$ which all contain $p_{1}, \ldots, p_{9}$. Since $c_{1}(L)^{2}=E \cdot E=9$, these cubics are disjoint outside $p_{1}, \ldots, p_{9}$ and therefore induce an elliptic fibration $\pi: X \rightarrow \mathbb{P}^{1}$ with one fiber isomorphic to $E$. Each point $p_{i}$ yields a section $\tau_{i}: \mathbb{P}^{1} \rightarrow X$. The fiberwise group law turns $X$ into a "group scheme" over the base scheme $\mathbb{P}^{1}$ with the section $\tau_{1}$ as neutral element. Now each of the sections $\tau_{i}$ yields an automorphism of $X$ which transfers the section $\tau_{1}$ into the section $\tau_{i}$. Since the section $\tau_{1}$ gives an exceptional curve on $X$ and since $\tau_{2}$ defines an automorphism of infinite order, we obtain, by repeated translation by $\tau_{2}$, a countable infinite family of exceptional curves which are evidently all defined over $k$ and all contain infinitely many $k$-rational points (they were obtained from blowing up $k$-rational points).

\section{An Example on $\mathbb{P}^{2}$ Blown Up at a Point}

In this section we give an example of a somewhat different sort which directly shows that the error term in Conjecture 2 cannot be replaced by an error term 
such as $\log h_{A}$.

Theorem 15. Let $\pi: X \rightarrow \mathbb{P}^{2}$ be the map obtained by blowing up $\mathbb{P}^{2}$ at a $\mathbb{Q}$-rational point $Q$. Let $D=\pi^{*}\left(L_{1}+L_{2}+L_{3}\right)$ be a divisor on $X$ where $L_{1}$, $L_{2}$, and $L_{3}$ are three lines on $\mathbb{P}^{2}$, defined over $\mathbb{Q}$, not containing $Q$. Let $K$ be the canonical divisor of $X$ and let $A$ be an ample divisor on $X$. Then for all $c_{1}, c_{2}>0$, the inequality

$$
m_{D, S}(P)+h_{K}(P)>c_{1} \log h_{A}(P)+c_{2}
$$

holds for a Zariski-dense set of points $P \in X(\mathbb{Q})$.

This theorem is essentially a combination of an observation of Silverman in [13] and a remark of Bugeaud, Corvaja, and Zannier in [4].

Proof. Without loss of generality, we may assume $Q=(1,1,1)$ and $L_{1}, L_{2}$, and $L_{3}$ are defined by $x=0, y=0$, and $z=0$, respectively. Let $S$ be a finite set of places of $\mathbb{Q}$ containing the infinite place. Let

$$
R=\left\{\pi^{-1}((u, v, 1)) \mid u, v \in \mathbb{Z}_{S}^{*} \cap \mathbb{Z},(u, v) \neq(1,1)\right\} .
$$

Note that $K_{X} \sim \pi^{*}(-3 L)+E$ where $L$ is a line on $\mathbb{P}^{2}$ and $E$ is the exceptional curve on $X$. By the definition of $R$ and $D$, clearly we have

$$
m_{D, S}(P)=h_{D}(P)+O(1), \quad \forall P \in R .
$$

So

$$
m_{D, S}(P)+h_{K_{X}}(P)=h_{D}(P)+h_{K_{X}}(P)+O(1)=h_{E}(P)+O(1), \quad \forall P \in R,
$$

since $D+K_{X} \sim E$. We now explicitly compute $h_{E}(P)$.

Lemma 16. If $P=\pi^{-1}((u, v, 1)) \in R$ then

$$
h_{E}(P)=\log \operatorname{GCD}(u-1, v-1)+O(1) .
$$

Proof. Let $\phi: X \rightarrow \mathbb{P}^{1}$ be the morphism induced by the rational map $\mathbb{P}^{2} \rightarrow \mathbb{P}^{1}$, $(x, y, z) \mapsto(x-z, y-z)$. Let $M$ be the line $y=z$ on $\mathbb{P}^{2}$. So $\phi^{*}((1,0))=\pi^{*} M-E$. By functoriality of heights, for $P=\pi^{-1}((u, v, 1)) \in R$ we get

$$
h_{\pi^{*} M-E}(P)=h_{(1,0)}(\phi(P))=\log \frac{\max \{|u-1|,|v-1|\}}{\operatorname{GCD}(u-1, v-1)} .
$$

Again, by functoriality, we have

$$
h_{\pi^{*} M}(P)=h_{M}(\pi(P))=\log \max \{|u-1|,|v-1|\}+O(1) .
$$

Therefore,

$$
h_{E}(P)=h_{\pi^{*} M}(P)-h_{\pi^{*} M-E}(P)+O(1)=\log \operatorname{GCD}(u-1, v-1)+O(1) .
$$


For fixed integers $a, b>1$, and an appropriate $S, a^{n}$ and $b^{n}$ are $S$-units for any integer $n$. So $\pi^{-1}\left(\left(a^{n}, b^{n}, 1\right)\right) \in R$ for all $n \in \mathbb{N}$. By Lemma 16, getting a lower bound for $h_{E}$ on these points is equivalent to getting a lower bound on $\operatorname{GCD}\left(a^{n}-1, b^{n}-1\right)$. Using Fermat's theorem, we may easily get a lower bound for this GCD if we can construct positive integers $n$ that are divisible by $p-1$ for many primes $p$. We use the following theorem.

Theorem 17 (Adleman, Pomerance, Rumely [1]). There exists a constant $C>$ 0 such that

$$
\#\{p: p \text { is prime, } p-1 \mid N\}>e^{C \log N / \log \log N}
$$

holds for infinitely many positive integers $N$.

Therefore, using Fermat's theorem and Theorem 17, we see that for any integers $a, b>1$,

$$
\operatorname{GCD}\left(a^{N}-1, b^{N}-1\right)>e^{e^{C \log N / \log \log N}}
$$

for infinitely many positive integers $N$ and some constant $C>0$. Let $A=\pi^{*} L_{3}$. Then $h_{A}\left(\pi^{-1}\left(\left(a^{N}, b^{N}, 1\right)\right)=N \max \{\log a, \log b\}\right.$. Thus translating (2) into heights gives

$$
h_{E}(P)>e^{C^{\prime} \log h_{A}(P) / \log \log h_{A}(P)}, \quad P=\pi^{-1}\left(\left(a^{N}, b^{N}, 1\right)\right),
$$

for infinitely many positive integers $N$ and some constant $C^{\prime}>0$.

If $a$ and $b$ are multiplicatively independent $\left(a^{n} \neq b^{m}\right.$ for any integers $m$ and $n)$ then it is easy to show that the set of points $\left\{\left(a^{N}, b^{N}, 1\right) \mid N \in I\right\}$ is Zariski-dense in $\mathbb{P}^{2}$ for any infinite set of positive integers $I$. Indeed, suppose $\sum_{i, j=1}^{n} c_{i j} a^{N i} b^{N j}=0$ for all $N$ in $I$. Since $a$ and $b$ are multiplicatively independent, the numbers $a^{i} b^{j}$ are distinct for all $i$ and $j$, and therefore there is a largest $a^{i} b^{j}$ with $c_{i j} \neq 0$ for $1 \leq i, j \leq n$. As $N \rightarrow \infty, N \in I$, clearly we get a contradiction in the equation.

So for $A=\pi^{*} L_{3}$, we see that (1) holds on a Zariski-dense subset of $X$. For arbitrary ample (or big) $A$, we finish by noting that since $\pi^{*} L_{3}$ is a big divisor, there exist positive constants $d_{1}$ and $d_{2}$ such that $h_{A}<d_{1} h_{\pi^{*} L_{3}}+d_{2}$ outside of a Zariski-closed subset of $X$ (see [14, Prop. 1.2.9(h)]).

\section{Acknowledgments}

We would like to thank Paul Vojta for allowing us to include Example 7 and for many helpful comments and suggestions on the paper.

\section{References}

[1] L. M. Adleman, C. Pomerance, and R. S. Rumely, On distinguishing prime numbers from composite numbers, Ann. of Math. (2) 117 (1983), no. 1, $173-206$. 
[2] W. P. Barth, K. Hulek, C. A. M. Peters, and A. Van de Ven, Compact complex surfaces, second ed., vol. 4, Springer-Verlag, Berlin, 2004.

[3] F. A. Bogomolov and Yu. Tschinkel, Density of Rational Points on Elliptic K3 Surfaces, Asian J. Math. 4 (2000), 351-368.

[4] Y. Bugeaud, P. Corvaja, and U. Zannier, An upper bound for the G.C.D. of $a^{n}-1$ and $b^{n}-1$, Math. Z. 243 (2003), no. 1, 79-84.

[5] R. Hartshorne, Algebraic geometry, Springer-Verlag, New York, 1977, Graduate Texts in Mathematics, No. 52.

[6] R. Kobayashi, Value distribution theory of holomorphic curves into complex projective algebraic varieties and geometric Diophantine problems, AMS/IP Studies in Advanced Mathematics 5 (1997), 105-115.

[7] S. Lang, Number theory. III. Diophantine geometry, Encyclopaedia of Mathematical Sciences, vol. 60, Springer-Verlag, Berlin, 1991.

[8] D. McKinnon, Vojta's main conjecture for blowup surfaces, Proc. Amer. Math. Soc. 131 (2003), no. 1, 1-12.

[9] R. Miranda and U. Persson, On extremal rational elliptic surfaces, Math. Z. 193 (1986), no. 4, 537-558.

[10] M. Nagata, On rational surfaces. II, Mem. Coll. Sci. Univ. Kyoto Ser. A Math. 33 (1960/1961), 271-293.

[11] R. Nevanlinna, Zur Theorie der meromorphen Funktionen, Acta Math. 46 (1925), 1-99.

[12] W. M. Schmidt, Diophantine approximation, Lecture Notes in Mathematics, vol. 785, Springer, Berlin, 1980.

[13] J. H. Silverman, Generalized greatest common divisors, divisibility sequences, and Vojta's conjecture for blowups, Monatsh. Math. 145 (2005), no. $4,333-350$.

[14] P. Vojta, Diophantine approximations and value distribution theory, Lecture Notes in Mathematics, vol. 1239, Springer-Verlag, Berlin, 1987.

[15] _ A refinement of Schmidt's subspace theorem, Amer. J. Math. 111 (1989), no. 3, 489-518.

[16] — My conjecture is false without the $\epsilon$, (1994), (letter to S. Lang).

[17]_, On Cartan's theorem and Cartan's conjecture, Amer. J. Math. 119 (1997), no. 1, 1-17. 